Research Paper

\title{
Dimethyloxaloylglycine Improves Angiogenic Activity of Bone Marrow Stromal Cells in the Tissue-Engineered Bone
}

\author{
Hao Ding*, Song Chen*, Wen-Qi Song, You-Shui Gao, Jun-Jie Guan, Yang Wang, Yuan Sun ${ }^{\bowtie}$ and \\ Chang-Qing Zhang ${ }^{\bowtie}$ \\ Department of Orthopedic Surgery, Shanghai Jiao Tong University Affiliated Sixth People's Hospital, Shanghai, China \\ *These authors provided equal contribution to this work.
}

$\triangle$ Corresponding authors: Chang-Qing Zhang (E-mail: zhangcq@sjtu.edu.cn), Department of Orthopedic Surgery, Shanghai Sixth People's Hospital, 600 Yishan Road, Shanghai, China; Yuan Sun (E-mail: david1979982@163.com), Department of Orthopedic Surgery, Shanghai Sixth People's Hospital, 600 Yishan Road, Shanghai, China.

(C) Ivyspring International Publisher. This is an open-access article distributed under the terms of the Creative Commons License (http://creativecommons.org/ licenses/by-nc-nd/3.0/). Reproduction is permitted for personal, noncommercial use, provided that the article is in whole, unmodified, and properly cited.

Received: 2014.0I.09; Accepted: 2014.02.08; Published: 2014.06.27

\begin{abstract}
One of the big challenges in tissue engineering for treating large bone defects is to promote the angiogenesis of the tissue-engineered bone. Hypoxia inducible factor-I $\alpha$ (HIF-I $\alpha$ ) plays an important role in angiogenesis-osteogenesis coupling during bone regeneration, and can activate a broad array of angiogenic factors. Dimethyloxaloylglycine (DMOG) can activate HIF-I $\alpha$ expression in cells at normal oxygen tension. In this study, we explored the effect of DMOG on the angiogenic activity of bone mesenchymal stem cells (BMSCs) in the tissue-engineered bone. The effect of different concentrations of DMOG on HIF-la expression in BMSCs was detected with western blotting, and the mRNA expression and secretion of related angiogenic factors in DMOG-treated BMSCs were respectively analyzed using QRT-PCR and enzyme linked immunosorbent assay. The tissue-engineered bone constructed with $\beta$-tricalcium phosphate $(\beta$-TCP) and DMOG-treated BMSCs were implanted into the critical-sized calvarial defects to test the effectiveness of DMOG in improving the angiogenic activity of BMSCs in the tissue-engineered bone. The results showed DMOG significantly enhanced the mRNA expression and secretion of related angiogenic factors in BMSCs by activating the expression of HIF-I $\alpha$. More newly formed blood vessels were observed in the group treated with $\beta$-TCP and DMOG-treated BMSCs than in other groups. And there were also more bone regeneration in the group treated with $\beta$-TCP and DMOG-treated BMSCs. Therefore, we believed DMOG could enhance the angiogenic activity of BMSCs by activating the expression of HIF-I $\alpha$, thereby improve the angiogenesis of the tissue-engineered bone and its bone healing capacity.
\end{abstract}

Key words: angiogenesis, bone marrow stromal cells, bone substitutes, dimethyloxaloylglycine, hypoxia inducible factor-1a

\section{Introduction}

Large bone defects, which caused by severe trauma, tumor resection or congenital deformities, were still difficult to treat in the clinic. The limited supply, the risk of rejection and the cost are serious limitations for autologous or allogeneic bone implantation to treat large bone defects [1,2]. Tissue- engineered bone is a new therapeutic strategy to repair large bone defects. Angiogenesis is a prerequisite step in bone tissue regeneration, and sufficient vascular supply is beneficial to bone repair and regeneration. Implantation of bone substitutes without adequate vascularity could lead to apoptosis and carti- 
lage formation [3]. Therefore, how to promote the angiogenesis of the tissue-engineered bone is an interesting issue of tissue engineering.

Hypoxia inducible factor-1a (HIF-1a) is a crucial mediator of the adaptive cells response to hypoxia, which plays an important role in angiogenesis-osteogenesis coupling during bone regeneration [4]. Previous studies showed HIF-1a could activate a broad array of genes and modulates cell proliferation, differentiation and pluripotency [5]. One of the major target genes of HIF-1a is vascular endothelial growth factor (VEGF), which is a potent mitogen for endothelial cells and plays an important role in neovascularization and angiogenesis [6]. Besides, HIF-1a can also activate the transcription of many other angiogenic genes in cells, such as SDF1, Ang-1, SCF, PLGF, bFGF, TGF- $\beta$ and ANGPT1 $[7,8]$. Previous studies have transduced the HIF-1a gene into mesenchymal stem cells (MSCs) with lentivirus vectors, and found HIF-1a transgenic MSCs had better angiogenic capacity, leading to better bone healing in large bone defects $[9,10]$. However, once transduced into cells, the HIF-1a gene will be overexpressed throughout the cell's life. The risks of lentivirus vectors, such as tumorigenesis, should also be carefully considered before the application in the clinic.

Dimethyloxaloylglycine (DMOG) is a small molecular drug, and is a cell permeable prolyl-4-hydroxylase inhibitor, which can stabilize the expression of HIF-1a in cells at normal oxygen tension [11]. Therefore, DMOG is expected to be an alternative strategy for enhancing HIF-1a expression in cells, which may be safely used in the clinic. In previous studies, by activating the expression of HIF-1a, DMOG has been successfully used to attenuate post-ischemic myocardial injury [12] and renal injury in remnant kidney [13], induce angiogenesis in ischemic skeletal muscles [14], and provide neuroprotection in a middle cerebral artery occlusion model [15]. In our previous study, we demonstrated that DMOG could improve the bone healing capacity of adipose-derived stem cells (ASCs) through enhancing its osteogenic differentiation and angiogenic potential [16]. In this study, we explored whether DMOG could increase the angiogenic activity of bone marrow stromal cells (BMSCs) in the tissue-engineered bone though activating the expression of HIF-1a in cells, and thereby improve the angiogenesis of the tissue-engineered bone.

\section{Materials and Methods}

\section{Isolation and culture of BMSCs}

Primary BMSCs were harvested from the bone marrow of F344 rats (Shanghai Animal Experimental
Center, Shanghai, China) according to the protocol reported previously [17]. All procedures adhered to the recommendations of the US Department of Health for the care and use of laboratory animals and were approved by the Ethics Committee of Shanghai Jiao Tong University. Briefly, both ends of the femur of 6-weeks-old F344 rats were cut off at the epiphysis, and the marrow was then flushed out with Dulbecco's modified Eagle's medium (DMEM) (GIBCO BRL, Grand Island, NY, USA) supplemented with $10 \%$ fetal bovine serum (FBS; Invitrogen, Carlsbad, CA, USA) and $200 \mathrm{U} / \mathrm{ml}$ of heparin (Sigma, St. Louis, MO, USA). Cells were cultured in DMEM containing 10\% FBS at $37^{\circ} \mathrm{C}$ in a humidified $5 \% \mathrm{CO} 2$ incubator. The culture medium was replaced every 3 days with non-adherent cells removed. When $90 \%$ confluence was reached, BMSCs were released from the culture substratum using $0.25 \%$ trypsin (Invitrogen, Carlsbad, CA, USA), and were then passaged at a 1:3 split. To avoid contamination of hematopoietic cells in earlier passages and differentiated cells in later passages, cells of four to six passages were used for the following experiments.

\section{Construction and transduction of HIF-I $\alpha$ shRNA}

To inhibit the expression of HIF-1a in BMSCs, the HIF-1a shRNA (Forward oligo: 5' TCCAGTTGAATCTTCAGATATTCAAGAGATATC TGAAGATTCAACTGG TTTTTT C 3', Reverse oligo: 5' TCGAGAAAAAACCAGTTGAATCTTCAGATA TCTCTTGAATATCTGAAGATTCAACTGGA 3', Invitrogen, Carlsbad, CA, USA) was constructed with pll3.7(Lentilox 3.7)-Zsgreen vector (addgene, cambridge, MA, USA). For transduction, BMSCs were plated on a 6-well plate (Corning, Tewksbury, MA, USA) at $1 \times 10^{5}$ cells and infected with the HIF-1a shRNA lentivirus at a multiplicity of infection (MOI) of 75 in the presence of $8 \mu \mathrm{g} / \mathrm{ml}$ polybrene (Sigma, St. Louis, MO, USA). The infection efficiency of the shHIF-1a lentivirus for BMSCs was reflected by the GFP-positive proportion of cells detected with flow cytometry, and the result showed that $92.3 \pm 2.6 \%$ was GFP-positive at $48 \mathrm{~h}$ after transduction.

\section{Western blotting analysis}

Western blotting analysis was performed to evaluate the influence of DMOG on the expression of HIF-1a in BMSCs. The cells were cultured in regular medium added with different concentrations of DMOG $(0,200,500,1000 \mu \mathrm{M})$ under normal oxygen conditions. After 12, 24 and $72 \mathrm{~h}$, total protein was harvested from the cultured cells according to standard protocols. Then the protein concentration was measured with a BCA protein assay kit (Thermo Sci- 
entific, Waltham, MA, USA). The cell lysates were separated on SDS-PAGE using $12 \%$ gels and transferred to nitrocellulose membranes. The membranes were then incubated with primary antibodies of HIF-1a (abcam, cambridge, MA, USA) at a 1:800 dilution overnight, followed by incubation with infrared-conjugated secondary antibodies at 1:10000 for 60 minutes at room temperature. The membranes were scanned in an Odyssey Scanner (Li-COR Biosciences, Lincoln, NE, USA), and bands were quantified with the Odyssey software V3.0. The protein levels were normalized against $\beta$-actin (abcam, cambridge, MA, USA). shHIF-1a BMSCs cultured in regular medium added with $1000 \mu \mathrm{M}$ DMOG were also performed as controls.

\section{Quantitative RT-PCR analysis}

Quantitative RT-PCR was carried out to detect the expression of related angiogenic genes in BMSCs at $0,1,3,7$ and 14 days after exposing to $1000 \mu \mathrm{M}$ DMOG. Total RNA was extracted using the TRIzol Plus RNA purification kit (Invitrogen, Carlsbad, CA, USA) according to the manufacturer's instructions. One microgram of pure RNA in a final volume of 20 $\mu \mathrm{l}$, as assessed spectrophotometrically using the A260/A280 ratio, was reverse transcribed using PrimeScriptTM RT reagent kit (Takara Bio, Shiga, Japan). Two microliters of the reverse transcription reaction were mixed with iQ SYBR Green super mix and amplified using iQ5 real-time system. The product was quantified using a standard curve and all values were normalized to GAPDH. Highly purified gene-specific primers for vascular endothelial growth factor (VEGF), stromal-derived factor 1 (SDF1), basic fibroblast growth factor (bFGF), placenta growth factor (PLGF) and GAPDH were synthesized commercially, and the primer pairs are listed in Table 1. All reactions were performed in triplicate.

\section{Enzyme linked immunosorbent assay (ELISA)}

The effect of DMOG on the secretion of related angiogenic factors from BMSCs were evaluated with ELISA. Normal BMSCs and shHIF-1a BMSCs were cultured in the regular medium added with $1000 \mu \mathrm{M}$ DMOG, and passaged as usual when reached to $90 \%$ confluence. After 0, 1, 3, 7 and 14 days, the cells were seeded into six-well plates at 300,000 cells/well, and cultured with the regular medium. After incubation for $24 \mathrm{~h}$, the medium was harvested and stored at $-80^{\circ} \mathrm{C}$. Then the protein secretions of VEGF, SDF1, bFGF and PLGF were analyzed using ELISA kits (Wuhan Xinqidi Biological Technology, Wuhan, China) according to the manufacturer's instructions. Normal BMSCs cultured in the regular medium without DMOG were also performed as controls.
Table I. Quantitative real-time polymerase chain reaction primer sequences.

\begin{tabular}{llll}
\hline Gene & Primer sequence $\left(5^{\prime}-3^{\prime}\right)(\mathrm{F} / \mathrm{R})$ & $\begin{array}{l}\text { Product } \\
\text { size }\end{array}$ & $\begin{array}{l}\text { Accession num- } \\
\text { ber }\end{array}$ \\
VEGF & $\begin{array}{l}\text { GGCTCTGAAACCATGAACTTTCT } \\
\text { GCAGTAGCTGCGCTGGTAGAC }\end{array}$ & 165 & NM_031836.2 \\
SDF1 & $\begin{array}{l}\text { ACCTCGGTGTCCTCTTGCTG } \\
\text { GATGTTTGACGTTGGCTCTGC }\end{array}$ & 172 & NM_001033883.1 \\
bFGF & $\begin{array}{l}\text { AAATCGCTATCTTGCTATGAAGGA } \\
\text { PATCGTTTCAGTGCCACATACC }\end{array}$ & 153 & NC_005101.2 \\
PLGF & $\begin{array}{l}\text { GGGAACAACTCAACAGAAATGG } \\
\text { GACTACAGCGACTCAGAAGGACA }\end{array}$ & 158 & NM_053595.2 \\
GAPDH ATGTTGTGATGGGCGTGAAGGTCT 174 & NM_017008.3 \\
& TCTGGGTGGCAGTGAT & & \\
\hline
\end{tabular}

\section{Animal experiments}

The $\beta$-tricalcium phosphate $(\beta$-TCP) scaffolds (Shanghai Bio-lu Biomaterials, Shanghai, China) was pie-shaped $(\Phi 5 \mathrm{~mm} \times 2 \mathrm{~mm} 3)$ and porous with an average pore size of $500 \mu \mathrm{m}$ and $75 \%$ porosity. Before attachment to the scaffolds, BMSCs were pretreated with $1000 \mu \mathrm{M}$ DMOG for $72 \mathrm{~h}$. Then cells at a density of $1.0 \times 10^{5}$ cells $/ \mathrm{ml}$ were slowly added to the $\beta$-TCP scaffolds until saturated, and were incubated with the scaffolds for at least $4 \mathrm{~h}$. The BMSCs-scaffolds constructs were subjected to scanning electron microscopy (SEM). To evaluate the in vivo angiogenic activity of DMOG-treated BMSCs, critical-sized calvarial defects models in rats were performed. In brief, following anesthesia with intraperitoneal pentobarbital (3 mg/100 g, Sigma, St. Louis, MO, USA), two 5-mm defects in the frontal parietal bone were carefully made using a trephine drill (Nouvag AG, Goldach, Switzerland). Then scaffolds with or without cells were implanted into the defects in the parietal bones.

These animals were randomly divided into four groups. Group I $(n=10)$ underwent implantation of $\beta$-TCP scaffolds alone. Group II $(n=10)$ underwent implantation of the tissue-engineered bone constructed with normal BMSCs and $\beta$-TCP. Group III $(n=10)$ underwent implantation of the tissue-engineered bone constructed with DMOG-treated BMSCs and $\beta$-TCP. Group IV $(n=10)$ underwent implantation of the tissue-engineered bone constructed with DMOG-treated shHIF-1a BMSCs and $\beta$-TCP.

\section{Microfil perfusion}

Eight weeks after surgery, these rats were euthanized and perfused with Microfil (Microfil MV-122, Flow Tech; Carver, MA, USA). In brief, the animals were anesthetized with intraperitoneal pentobarbital (3 mg/100 g, Sigma, St. Louis, MO, USA). The rib cage was opened, the descending aorta was clamped, and the inferior vena cava was incised. Then $20 \mathrm{ml}$ of heparinized saline (Shanghai No.1 Biochemical \& Pharmaceutical Co., Ltd, Shanghai, China) and 
$20 \mathrm{ml}$ of Microfil were respectively perfused into the left ventricle with an angiocatheter. Subsequently, the animals were stored at $4^{\circ} \mathrm{C}$ for $1 \mathrm{~h}$ to ensure polymerization of the contrast agent.

\section{MicroCT Scanning}

After microfil perfusion, the parietal bone of the animals were harvested and fixed in paraformaldehyde $(4 \%)$. Then they were scanned by microCT (SkyScan 1076, Kontich, Belgium) with a high-resolution system to evaluate new bone formation in the calvarial defect area. Briefly, the samples were scanned continuously at a resolution of 25 $\mu \mathrm{m}$ per voxel. The images were segmented with a low pass filter to remove noise, and bone tissue was defined at a threshold of 800 . The parameters of bone volume (BV), tissue volume (TV) and bone mineral density (BMD) of the calvarial defect area were calculated using the analysis software. The ratio of BV to TV indicates the relative amount of new bone in the calvarial defect area. BMD represented the mean bone density of the calvarial defect area.

Half of the samples for each group $(n=5)$ were then decalcified with ethylene diamine tetra acetic acid (EDTA, 10\%, PH 7.4), and scanned again by microCT to measure the angiogenesis in the calvarial defect area. The scan was performed at a resolution of $18 \mu \mathrm{m}$ per voxel with $1024 \times 1024$ pixel image matrix. Noise was removed with a low pass Gaussian filter. The blood vessels filled with Microfil were included with semi-automatically drawn contour at each two-dimensional section by built-in "Contouring Program" for automatic reconstruction of 3-D image of vasculature in the samples. The axial slices through the samples were then visualized. The number of vessels in the defects area and the vessels volume represented by the voxels counted in the specified microfil range were calculated.

\section{Histological observation}

The other five samples of each group were dehydrated in alcohol from $70 \%$ to $100 \%$, and embedded in polymethylmethacrylate (PMMA, Sinopharm Chemical Reagent Co., Ltd, Shanghai, China). Then the samples were cut with a microtome (Leica, Germany) at the sagittal section, which represented the central area of the bone defect. The sections were polished to a final thickness of approximately $40 \mathrm{~mm}$, and stained with van Gieson's picrofuchsin (Sinopharm Chemical Reagent Co., Ltd, Shanghai, China) according to standard procedures [18]. Newly formed bone showed red, and the TCP scaffolds showed black. To evaluate new bone formation, the surface areas of the newly formed bone were calculated based on the entire defect area, using a Leica digital imaging system and NIH image analysis software.

\section{Immunohistochemistry}

After microCT, the decalcified samples were embedded in paraffin, and coronal cross sections at 5 $\mu \mathrm{m}$ were made at the central area of the defect. To evaluate the new blood vessels formation, immunohistochemistry for CD31 (ab24590, abcam, 1:200) was then performed in the sections. Briefly, the sections were dewaxed and rehydrated, and treated with antigen retrieval. The primary antibody were diluted in $5 \%$ BSA/TBS and applied to the sections at $4^{\circ} \mathrm{C}$ overnight. Subsequently, the biotinylated secondary antibody and ABC complex were applied, and DAB substrate was used to stain the sections. Finally, the sections were treated with hematoxylin and mounted. The results were analyzed with a light microscope. Blood vessels were defined with the positive CD31 stain and their typical round or oval structure.

\section{Statistical analysis}

Statistical analysis was performed using SPSS 12.0 software for Windows. All of the in vitro experiments were performed in triplicate and data were presented as mean \pm SD. Statistical significance was assessed using ANOVA with a SNK post-hoc analysis, where P-values $<0.05$ were considered statistically significant.

\section{Results \\ DMOG promotes expression of HIF-I $\alpha$ in BMSCs}

To evaluate the influence of DMOG on the expression of HIF-1a in BMSCs, the protein levels of HIF-1a in BMSCs treated with different concentrations of DMOG was detected by western blotting. The results showed the level of HIF-1a protein was enhanced in response to DMOG treatment in a dose-dependent manner. However, there was no notable increase in shHIF-1a BMSCs treated with 1000 $\mu \mathrm{M}$ DMOG (Figure 1A). After treatment with DMOG for $24 \mathrm{~h}$, the expression of HIF-1a in BMSCs increased to stable levels, which were approximately 2-, 4- and 5-fold for 200, 500 and $1000 \mu$ M DMOG, respectively. And the expression levels of HIF-1a in BMSCs treated with DMOG for $72 \mathrm{~h}$ had no significant difference compared to that treated with DMOG for $24 \mathrm{~h}$ (Figure 1B).

\section{DMOG enhances mRNA expression of angiogenic factors in BMSCs}

The mRNA levels of related angiogenic genes in BMSCs exposing to DMOG were detected using qRT-PCR in vitro. In DMOG-treated BMSCs, the ex- 
pression of VEGF, SDF1, bFGF and PLGF were all markedly increased compared to that in normal BMSCs (Figure 2). The increase in the expression of these angiogenic genes began at day 1 and reached to a stable level at day 3 . Then mRNA expression of these genes maintained at a high level from day 3 to

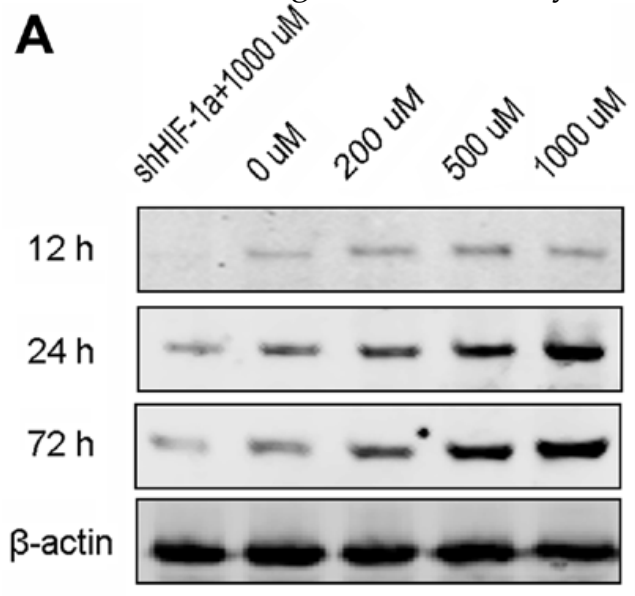

day 14. The mRNA levels of these factors in shHIF-1a BMSCs treated with DMOG remained at low levels from day 0 to day 14, which indicated DMOG enhanced mRNA expression of related angiogenic factors through activating the expression of HIF-1a.

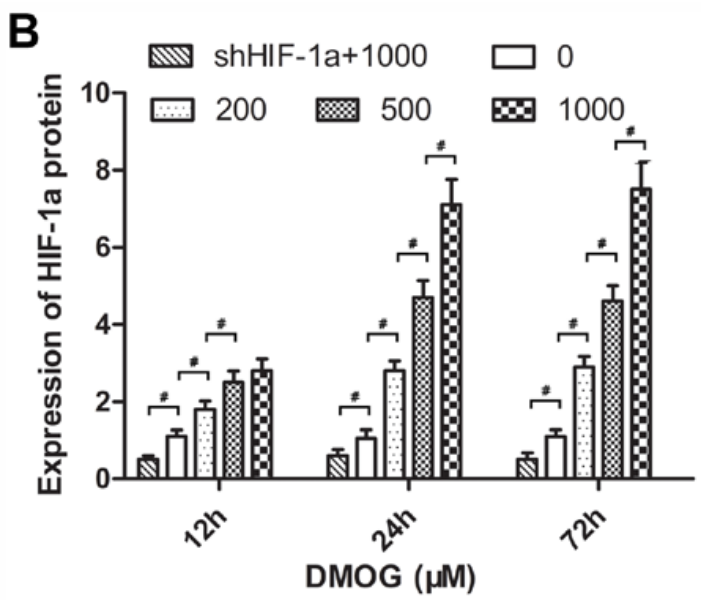

Figure I. Effect of DMOG on the expression of HIF-I $\alpha$ in BMSCs. (A) Western blotting showed that DMOG significantly enhanced the protein levels of HIF-I $\alpha$ in BMSCs. (B) The analysis suggested that the expression of HIF-I $\alpha$ in BMSCs was increased by DMOG in a dose-dependent manner, and there was no notable increase in shHIF-I $\alpha$ BMSCs treated with $1000 \mu$ M DMOG. (\#, significant difference between the two groups, $p<0.05$ )
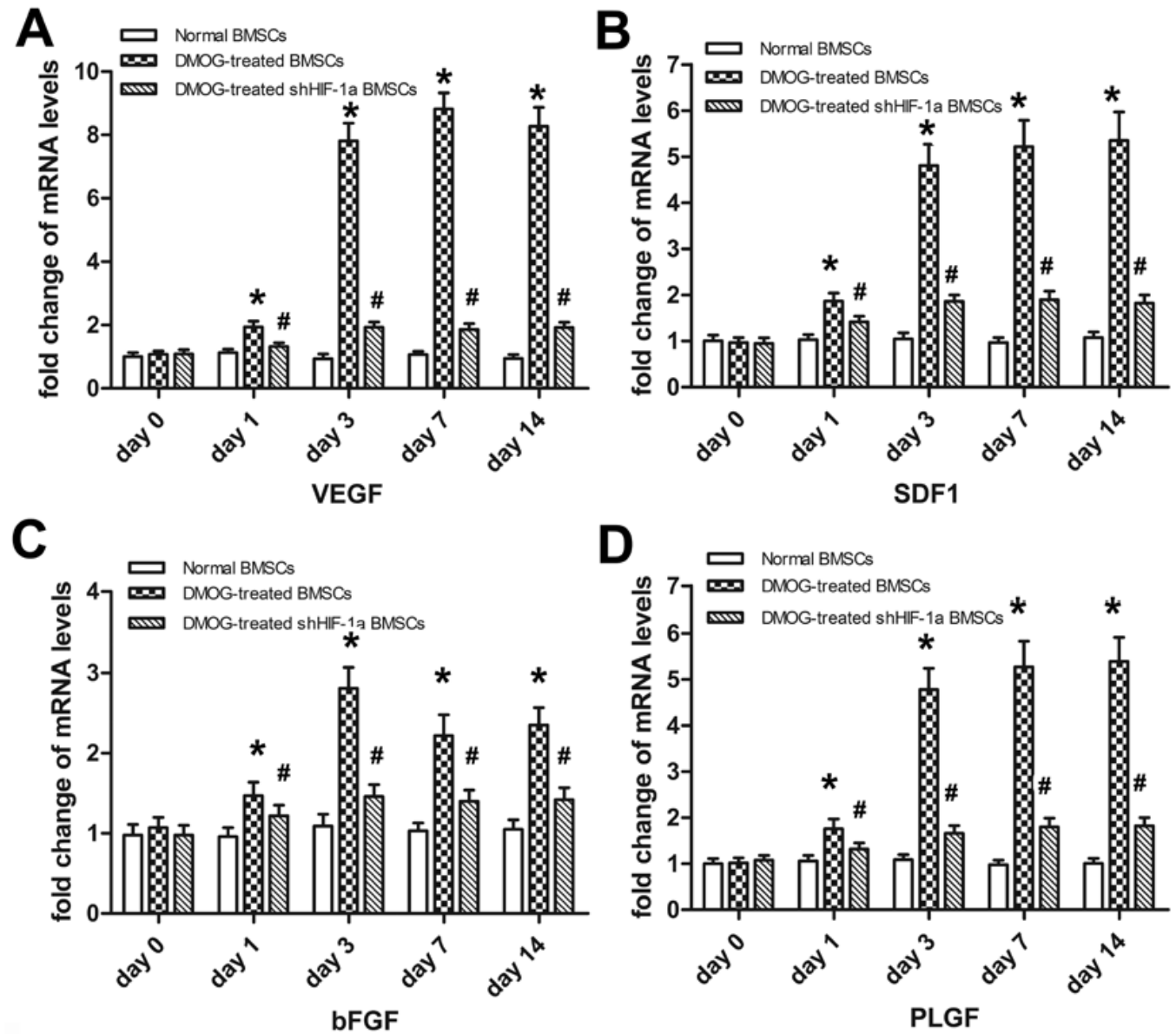

Figure 2. The effect of DMOG on the mRNA expression levels of related angiogenic genes in BMSCs were detected using qRT-PCR. The transcriptional levels of VEGF (A), SDFI (B), bFGF (C) and PLGF (D) in DMOG-treated BMSCs were all markedly increased compared to that in normal BMSCs from day I to day 3, and maintained at a high level from day 3 to day 14 . The increase was not significant in shHIF-I a BMSCs treated with DMOG. $(*$, significant difference between normal BMSCs and DMOG-treated BMSCs, $\mathrm{P}$ $<0.05$; \#, significant difference between DMOG-treated BMSCs and DMOG-treated shHIF-Ia BMSCs, $\mathrm{p}<0.05$ ) 


\section{DMOG enhances secretion of angiogenic factors from BMSCs}

To further detect the effect of DMOG on the angiogenic activity of BMSCs, the levels of related angiogenic factors secreted from BMSCs were analyzed by ELISA (Figure 3). VEGF production in BMSCs treated with DMOG markedly increased from day 1 to day 3 and was then maintained at a high level until day 14, which was obviously higher than VEGF production in normal BMSCs. However, VEGF production in shHIF-1a BMSCs treated with DMOG maintained at a lower level and did not change significantly from day 0 to day 14 . Other angiogenic factors, such as SDF1, bFGF and PLGF, showed the same pattern as VEGF. Taken together, these data supported that DMOG could enhance the secretion of related angiogenic factors from BMSCs in vitro, which was consistent with the qRT-PCR results.

\section{MicroCT analysis}

Before decalcification, the samples were scanned by microCT to evaluate new bone formation in the calvarial defect area. From the sagittal view, the new bone formation in Group III was more than that in other three groups (Figure 4A). A quantitative analysis showed that BV/TV of Group III was markedly higher than that of Group II, which was higher than that of Group I. However, BV/TV of Group IV was obviously lower than that of Group III (Figure 4B). Additionally, BMD of the four groups showed the same pattern as for BV/TV (Figure 4C). After decalcification, the samples were scanned again by microCT to evaluate the angiogenesis of the tissue-engineered bone in the calvarial defect area of each group. The reconstructions of the three-dimensional microCT images directly showed new blood vessels formation in the bone defects. Larger vascular network area was observed in Group III, which appeared significantly denser compared to other three groups (Figure 5A). Quantification analysis showed the number and total volume of blood vessels in the Group III were more than those in Group I, II and IV. The number and total volume of blood vessels in Group II were similar to those in Group IV, and more than those in Group I (Figure 5B,C).
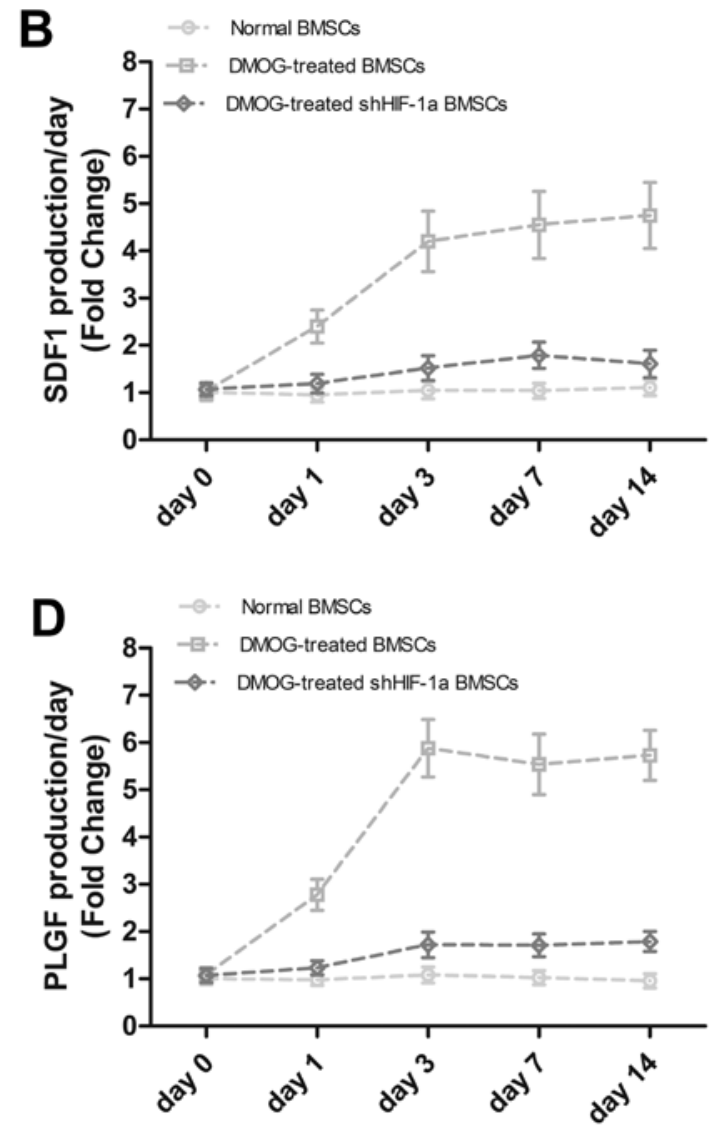

Figure 3. The effect of DMOG on the secretion of related angiogenic factors from BMSCs were analyzed by ELISA. The production of VEGF (A), SDFI (B), bFGF (C) and PLGF (D) secreted from normal BMSCs, DMOG-treated BMSCs and DMOG-treated shHIF-I $\alpha$ BMSCs were quantified. 
A B-TCP alone

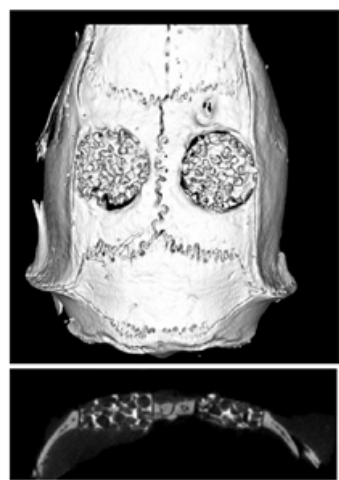

B

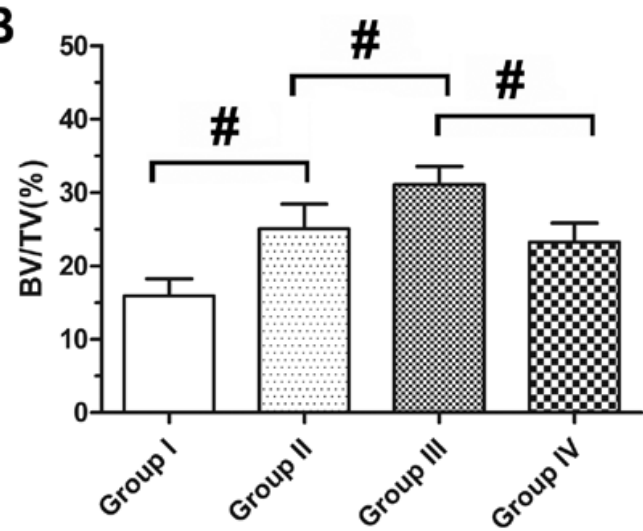

DMOG-treated

shHIF-1a BMSCs
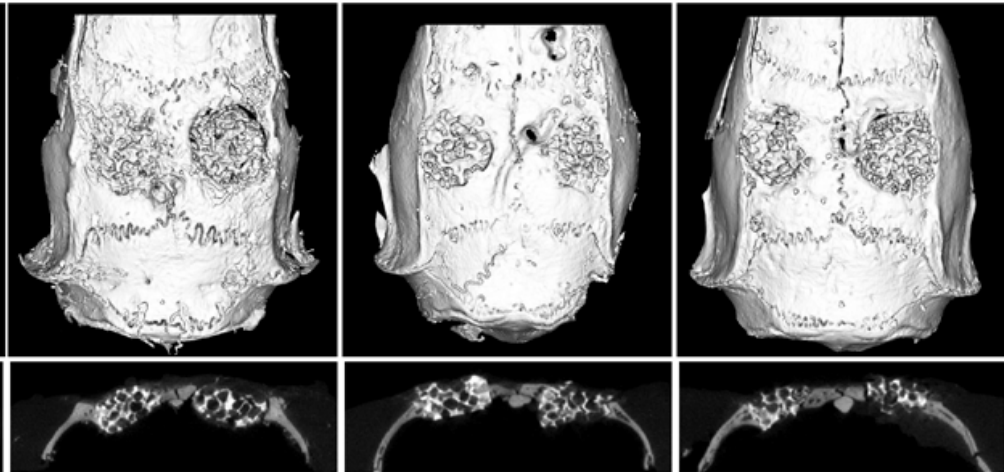

C

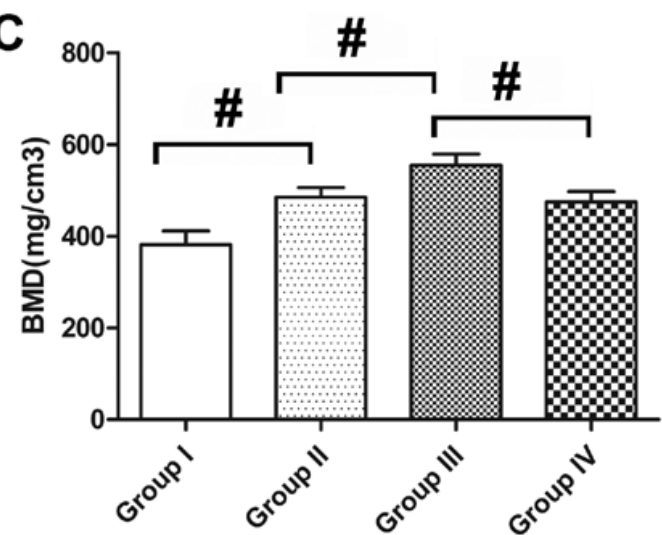

Figure 4. Bone regeneration in the defect area was evaluated using Micro-CT at 8 weeks after implantation with the tissue-engineered bone. (A) Micro-CT images of calvarial defects taken at 8 weeks after implantation. (B, C) Quantitative analysis showed bone mineral density (BMD) and bone volume/tissue volume (BV/TV) of the newly formed bone in the defects area of each group. (\#, significant difference between the two groups, $\mathrm{p}<0.05$ )

A

\section{B-TCP alone}

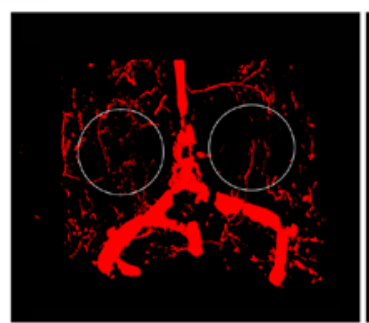

$\mathbf{B}$

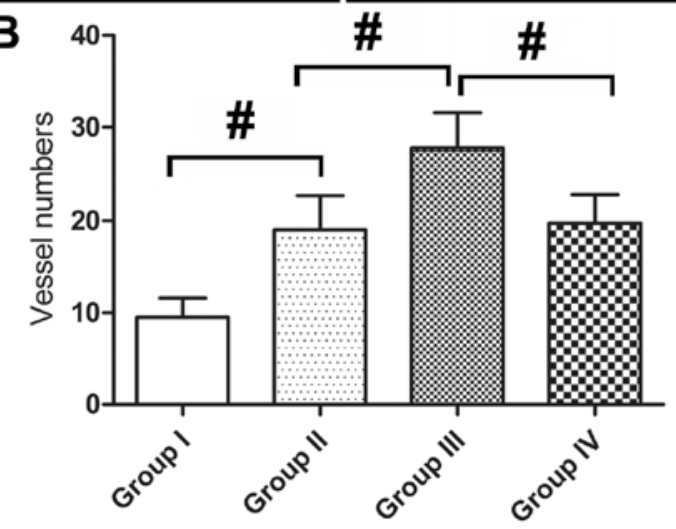

Normal BMSCs

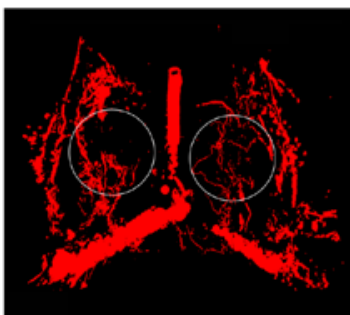

C

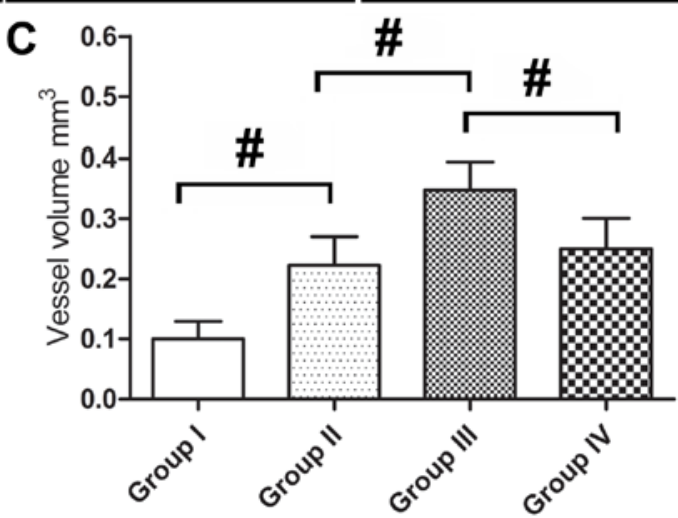

Figure 5. Angiogenesis in the defect area was evaluated using Micro-CT at 8 weeks after implantation with the tissue-engineered bone. (A) Representative images of micro-CT reconstructed 3D microangiography of the defect areas, which were evaluated as the region of interest (ROI) within the white frame. (B, C) Quantitative analysis showed the numbers and volumes of newly formed blood vessels in each group. (\#, significant difference between the two groups, $p<0.05$ ) 


\section{Histological analysis and immunohistochemistry}

Based on histological observation, the percentage of new bone area were $8.29 \pm 2.58 \%$ in Group I, $22.14 \pm 3.05 \%$ in Group II, $30.62 \pm 3.45 \%$ in Group III and $21.57 \pm 3.11 \%$ in Group IV (Figure 6). Immunohistochemistry for CD31 showed there were more new

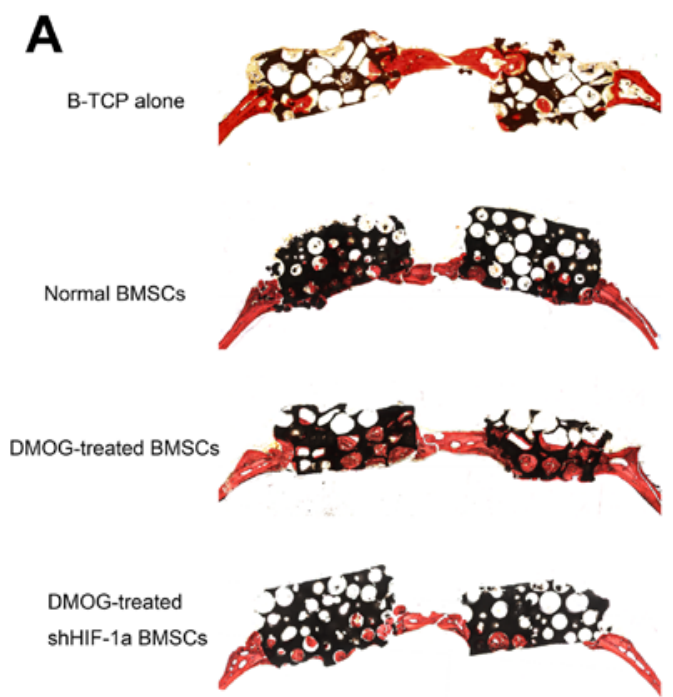

vessels formation in Group III than in Group II and IV, and nearly no blood vessels formed in Group I (Figure 7). The quantification analysis showed there were $0.8 \pm 0.3$ new vessels per field in Group I, $4.1 \pm 1.3$ in Group II, 7.2 \pm 1.6 in Group III and 3.7 \pm 1.1 in Group IV. The microCT results were further supported by histological analysis and immunohistochemistry.

B

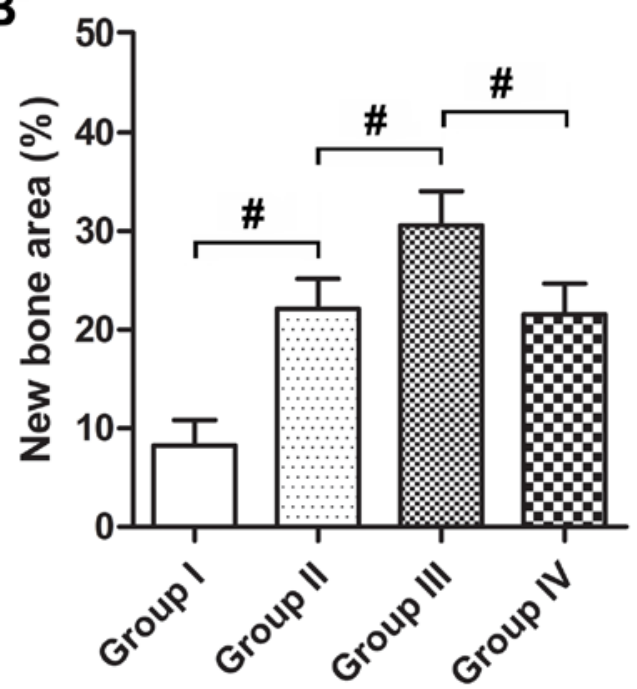

Figure 6. Histological analysis of bone regeneration in the defect area of each group. (A) Representative histological photomicrograph of bone regeneration in the defect area. (B) Histomorphometric analysis showed that there were more new bone formation in the defect area of Group III than that of Group II and IV, and there was nearly no bone regeneration in Group I. (\#, significant difference between the two groups, $\mathrm{p}<0.05$ )

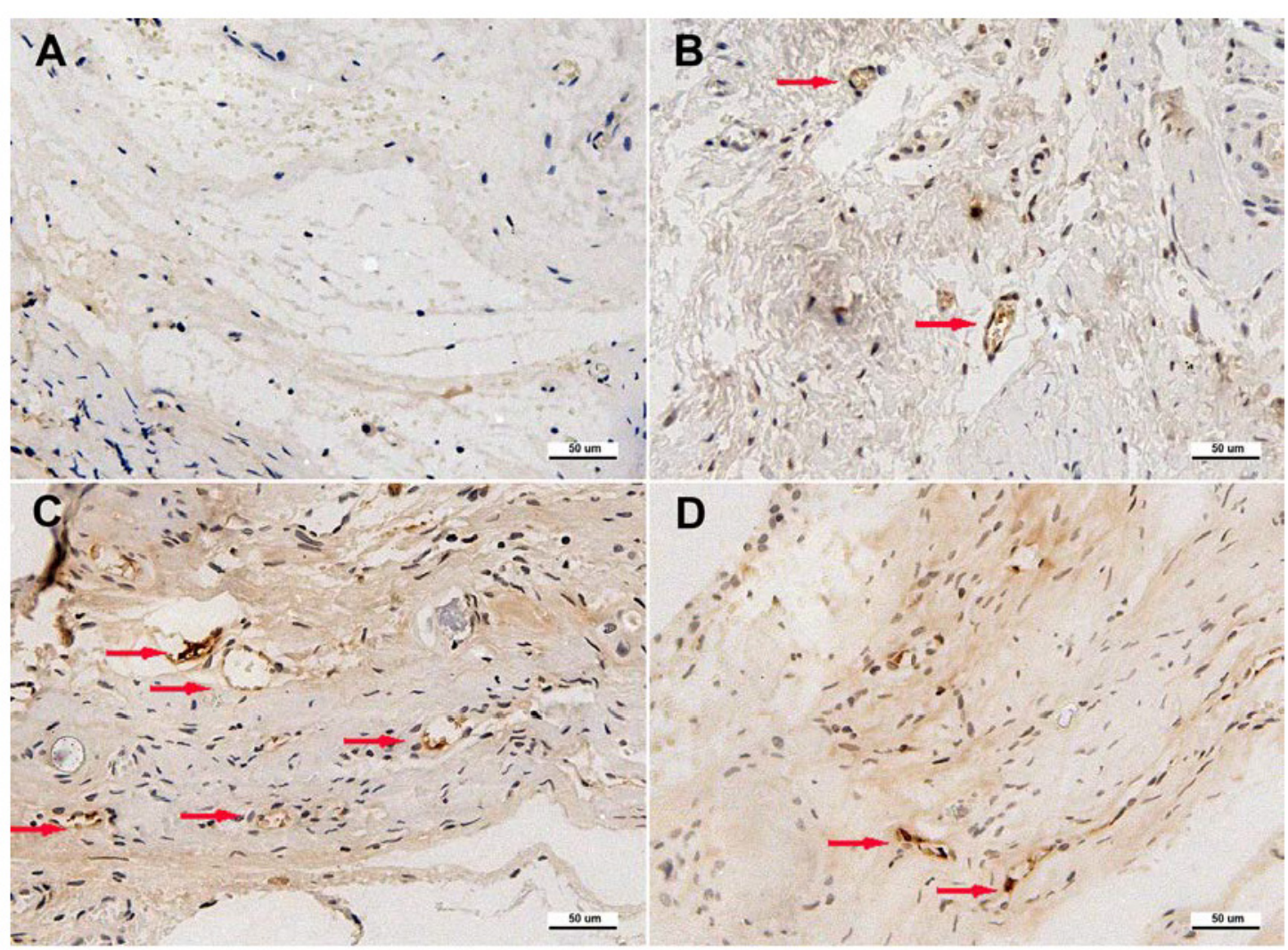

Figure 7. Immunohistochemical analysis of $C D 3 \mid$ in the defect area of each group at 8 weeks after implantation. Blood vessels were defined with the positive CD3I stain and their typical round or oval structure. Immunohistochemistry for CD3I showed there were more newly formed blood vessels (red arrows) in Group III (C) than in other three groups $(A, B$ and $D)$. (Scale bars $=50 \mu \mathrm{m}$.) 


\section{Discussion}

In this study, we explored the effect of DMOG on the angiogenic activity of BMSCs in the tissue-engineered bone constructed with BMSCs and $\beta$-TCP. The artificial bone substitutes such as tricalcium phosphate (TCP), hydroxyapatite and coralline scaffolds are promising therapeutic approaches for bone regeneration in large bone defects. However, researchers found the artificial bone substitutes alone are insufficient to repair large bone defects [19]. Angiogenesis is often limited to the periphery of these bone substitutes as they lack adequate capability to induce new vessels formation. Tissue engineering studies have explored the use of various cells in combination with these scaffolds to construct the tissue-engineered bone, thereby improve their angiogenic activities.

BMSCs are characterized as undifferentiated cells and possess a mesodermal differentiation potential, which are widely used as an attractive cell source for the regeneration of bone tissues [20, 21]. In the tissue-engineered bone, they not only directly differentiate into endothelial cells to participate in new blood vessels formation in the bone defect area [22, 23], but also secret angiogenic factors to promote local angiogenesis [24, 25]. This study showed there were obviously more new blood vessels formation in the normal BMSCs group than in the only $\beta$-TCP group, which demonstrated that the tissue-engineered bone had better angiogenic capacity than the artificial bone. However, limited numbers of functional cells could be seeded on the bone substitutes, so modified BMSCs with improved angiogenic activity will be greatly beneficial to the angiogenesis of the tissue-engineered bone.

Angiogenic activity of MSCs is regulated by various signaling pathways, which include bFGF, IGF-1, prostaglandins E1 and E2, TGF- $\beta$ and endothelin-1. All of them are associated with the HIF-1 pathway [26]. HIF-1 is an oxygen-sensitive complex, which is composed of HIF-1a and HIF-1 $\beta$. Under normoxic conditions, HIF-1a is hydroxylated by the enzyme HIF-PH, causing the ubiquitylation and degradation of HIF-1a. DMOG is a cell permeable prolyl-4-hydroxylase inhibitor, which can upregulate the protein level of HIF-1a post-transcriptionally under normoxic conditions. In this study, western blotting demonstrated that the expression levels of HIF-1a in BMSCs could be obviously upregulated when cells were exposed to DMOG, and the increase was dose-dependent. Therefore, after BMSCs exposing to DMOG, HIF-1a will be accumulated in cells, and then bound to HIF-1 $\beta$. The HIF-1 complex then activates the expression of various angiogenic genes.
The results of qRT-PCR in this study showed DMOG treatment could significantly enhance the transcriptional levels of VEGF, SDF1, bFGF and PLGF. Of them, VEGF is the most important angiogenic factor and has the most significant biological activity to promote new blood vessels formation [27]. The slow and continued release of VEGF is thought to be crucial for angiogenesis during bone regeneration [28, 29]. SDF1 plays an important role in recruiting endothelial progenitor cells (EPCs) to the ischemia area, and activation of the SDF1/CXCR4 axis has a direct effect on vascular endothelial cells to promote blood vessels growth [30]. bFGF is believed to have an more profound effect on blood vessels growth than VEGF at the early time point of the revascularization process [31]. PLGF has been reported to have a comparable effect to that of VEGF and bFGF on the microvascular endothelium, which could stimulate the migration of endothelial cells in vitro and induce angiogenesis in vivo [32].

The increase of the transcriptional levels of these genes was also significantly inhibited in shHIF-1a BMSCs, which indicated that DMOG enhanced the expression of these genes through activating HIF-1a expression in cells. ELISA for these angiogenic factors, which indicated the secretion of these factors from BMSCs, showed the same tendency as the qRT-PCR results. Angiogenesis is a complex process, which needs the involvement of multiple angiogenic factors. Researchers have reported that the use of a single factor was insufficient to form functional vascular structures [33]. All of the above factors take part in the process of angiogenesis, and the cooperation of these factors would have a better result in promoting new blood vessels formation [32, 34, 35].

To test the effectiveness of DMOG in improving the angiogenic activity of BMSCs in the tissue-engineered bone in vivo, $\beta$-TCP with DMOG-treated BMSCs were implanted into the critical-sized calvarial defects in rats. Micro-CT examination showed there were more new blood vessels observed in the DMOG-treated BMSCs group than in the normal BMSCs group, which demonstrated that DMOG could enhance angiogenesis of the tissue-engineered bone in vivo. The vessels formation was less in the DMOG-treated shHIF-1a BMSCs group than that in the DMOG-treated BMSCs group. This might be due to DMOG enhanced angiogenesis of the tissue-engineered bone through activating the HIF-1a expression in BMSCs, and DMOG played no role in the shHIF-1a BMSCs. The result of immunohistochemistry for CD31 also supported the above point of view.

There is a close association between angiogenesis and osteogenesis in bone regeneration, and affluent 
blood flow is an important favorable element for new bone formation [36, 37]. Orchestration of bone formation is closely linked to the crosstalk of endothelial cells and osteoblasts [38]. VEGF is believed to be an important mediator of this crosstalk. It is primarily released by osteoblasts, and its action is conducted by endothelial cells [39]. Endothelial cells could increase alkaline phosphatase activity and collagen I synthesis of BMSCs via gap junction communication, and stimulate osteoprogenitor cell differentiation, thereby improve osteogenesis $[40,41]$. The direct osteogenic effect of VEGF on osteoblasts may also play an important role in bone regeneration [36]. So there was more bone regeneration observed in the defect area of the DMOG-treated BMSCs group than that of other groups in this study.

In this study, we mainly explored the effect of DMOG on the angiogenesis of the tissue-engineered bone. However, the effect of DMOG on the angiogenesis of the biomaterial alone were also deserved to be studied. Wu et al had loaded DMOG on mesoporous bioactive glass scaffolds, found the release of DMOG from the scaffolds could improve angiogenesis and osteogenesis of nearby BMSCs [42]. We thought DMOG alone also had an effect on angiogenesis of the biomaterial which can release DMOG continuously. However, the effect of DMOG alone on the angiogenesis of such biomaterial in vivo is also need to be explored in the future. In our previous study, we demonstrated that DMOG could inhibit MSCs death induced by serum deprivation in a dose-dependent manner in vitro [16]. So we thought DMOG could enhance MSCs survival in vivo, which still need to be demonstrated by the further study in vivo.

In summary, this study demonstrated DMOG could increase the expression and secretion of some angiogenic factors in BMSCs in vitro by stabilizing the expression of HIF-1a in cells, and DMOG could improve the angiogenesis of the tissue-engineered bone through enhancing the angiogenic activity of BMSCs. The results provide an evidence for the further application of DMOG in improving angiogenesis and promoting bone regeneration in bone tissue engineering.

\section{Acknowledgments}

This work was supported by the National Natural Science Foundation of China (81101368, 81272003 and 81371962). We thank Professor Chuan-Gui Wang and his student Chen Hu (Shanghai Key Laboratory of Regulatory Biology, School of life Sciences, East China Normal University) for their valuable suggestions and technical advice.

\section{Abbreviations}

HIF-1a: Hypoxia inducible factor-1a; VEGF: vascular endothelial growth factor; Ang-1: Angiotension-1; SCF: Stem cell factor; TGF- $\beta$ : transforming growth factor- $\beta$; MSC:, mesenchymal stem cells; DMOG: Dimethyloxaloylglycine; ASCs: adipose-derived stem cells; BMSCs: bone marrow stromal cells; DMEM: Dulbecco's modified Eagle's medium; FBS: fetal bovine serum; SDF1: stromal-derived factor 1; bFGF: basic fibroblast growth factor; PLGF: placenta growth factor; $\beta$-TCP: $\beta$-tricalcium phosphate; BV: bone volume; TV: tissue volume; BMD: bone mineral density; EDTA: ethylene diamine tetra acetic acid; PMMA: polymethylmethacrylate; TCP: tricalcium phosphate.

\section{Competing Interests}

The authors have declared that no competing interest exists.

\section{References}

1. Bauer TW, Muschler GF. Bone graft materials. An overview of the basic science. Clin Orthop Relat Res. 2000;: 10-27.

2. Oreffo RO, Triffitt JT. Future potentials for using osteogenic stem cells and biomaterials in orthopedics. Bone. 1999; 25: 5S-9S.

3. Muschler GF, Nakamoto C, Griffith LG. Engineering principles of clinical cell-based tissue engineering. J Bone Joint Surg Am. 2004; 86A: 1541-58.

4. Riddle RC, Khatri R, Schipani E, Clemens TL. Role of hypoxia-inducible factor-1alpha in angiogenic-osteogenic coupling. J Mol Med (Berl). 2009; 87: 583-90.

5. Mazumdar J, Dondeti V, Simon MC. Hypoxia-inducible factors in stem cells and cancer. J Cell Mol Med. 2009; 13: 4319-28.

6. Ferrara N. Molecular and biological properties of vascular endothelial growth factor. J Mol Med (Berl). 1999; 77: 527-43.

7. Kelly BD, Hackett SF, Hirota K, Oshima Y, Cai Z, Berg-Dixon S, Rowan A, Yan $Z$, Campochiaro PA, Semenza GL. Cell type-specific regulation of angiogenic growth factor gene expression and induction of angiogenesis in nonischemic tissue by a constitutively active form of hypoxia-inducible factor 1 . Circ Res. 2003; 93: 1074-81.

8. Ceradini DJ, Kulkarni AR, Callaghan MJ, Tepper OM, Bastidas N, Kleinman ME, Capla JM, Galiano RD, Levine JP, Gurtner GC. Progenitor cell trafficking is regulated by hypoxic gradients through HIF-1 induction of SDF-1. Nat Med. 2004; 10: 858-64

9. Zou D, Zhang Z, He J, Zhang K, Ye D, Han W, Zhou J, Wang Y, Li Q, Liu X, Zhang $X$, Wang S, Hu J, Zhu C, Zhang W, zhou Y, Fu H, Huang Y, Jiang X. Blood vessel formation in the tissue-engineered bone with the constitutively active form of HIF-1alpha mediated BMSCs. Biomaterials. 2012; 33: 2097-108.

10. Ding H, Gao YS, Hu C, Wang Y, Wang CG, Yin JM, Sun Y, Zhang CQ. HIF-1alpha transgenic bone marrow cells can promote tissue repair in cases of corticosteroid-induced osteonecrosis of the femoral head in rabbits. PLoS One. 2013; 8: e63628.

11. Jaakkola P, Mole DR, Tian YM, Wilson MI, Gielbert J, Gaskell SJ, von Kriegsheim A, Hebestreit HF, Mukherji M, Schofield CJ, Maxwell PH, Pugh CW, Ratcliffe PJ. Targeting of HIF-alpha to the von Hippel-Lindau ubiquitylation complex by O2-regulated prolyl hydroxylation. Science. 2001; 292: 468-72.

12. Ockaili R, Natarajan R, Salloum F, Fisher BJ, Jones D, Fowler AA, 3rd, Kukreja RC. HIF-1 activation attenuates postischemic myocardial injury: role for heme oxygenase- 1 in modulating microvascular chemokine generation. Am J Physiol Heart Circ Physiol. 2005; 289: H542-8.

13. Song YR, You SJ, Lee YM, Chin HJ, Chae DW, Oh YK, Joo KW, Han JS, Na KY. Activation of hypoxia-inducible factor attenuates renal injury in rat remnant kidney. Nephrol Dial Transplant. 2010; 25: 77-85.

14. Milkiewicz M, Pugh CW, Egginton S. Inhibition of endogenous HIF inactivation induces angiogenesis in ischaemic skeletal muscles of mice. J Physiol. 2004; 560: 21-6.

15. Nagel S, Papadakis M, Chen R, Hoyte LC, Brooks KJ, Gallichan D, Sibson NR, Pugh C, Buchan AM. Neuroprotection by dimethyloxalylglycine following permanent and transient focal cerebral ischemia in rats. J Cereb Blood Flow Metab. 2011; 31: 132-43.

16. Ding H, Gao YS, Wang Y, Hu C, Sun Y, Zhang C. Dimethyloxalylglycine increases the bone healing capacity of adipose-derived stem cells by promot- 
ing osteogenic differentiation and angiogenic potential. Stem Cells Dev 2013; [Epub ahead of print]

17. Maniatopoulos C, Sodek J, Melcher AH. Bone formation in vitro by stromal cells obtained from bone marrow of young adult rats. Cell Tissue Res. 1988; 254: 317-30.

18. Rohrer MD, Schubert CC. The cutting-grinding technique for histologic preparation of undecalcified bone and bone-anchored implants. Improvements in instrumentation and procedures. Oral Surg Oral Med Oral Pathol. 1992; 74: 73-8.

19. Louisia S, Stromboni M, Meunier A, Sedel L, Petite H. Coral grafting supplemented with bone marrow. J Bone Joint Surg Br. 1999; 81: 719-24.

20. Deng Y, Wu S, Zhou H, Bi X, Wang Y, Hu Y, Gu P, Fan X. Effects of a miR-31, Runx2, and Satb2 regulatory loop on the osteogenic differentiation of bone mesenchymal stem cells. Stem Cells Dev. 2013; 22: 2278-86.

21. Deng Y, Zhou H, Zou D, Xie Q, Bi X, Gu P, Fan X. The role of miR-31-modified adipose tissue-derived stem cells in repairing rat critical-sized calvarial defects. Biomaterials. 2013; 34: 6717-28.

22. Miranville A, Heeschen C, Sengenes C, Curat CA, Busse R, Bouloumie A. Improvement of postnatal neovascularization by human adipose tissue-derived stem cells. Circulation. 2004; 110: 349-55.

23. Scherberich A, Galli R, Jaquiery C, Farhadi J, Martin I. Three-dimensional perfusion culture of human adipose tissue-derived endothelial and osteoblastic progenitors generates osteogenic constructs with intrinsic vascularization capacity. STEM CELLS. 2007; 25: 1823-9.

24. Kinnaird T, Stabile E, Burnett MS, Shou M, Lee CW, Barr S, Fuchs S, Epstein SE. Local delivery of marrow-derived stromal cells augments collateral perfusion through paracrine mechanisms. Circulation. 2004; 109: 1543-9.

25. Beckermann BM, Kallifatidis G, Groth A, Frommhold D, Apel A, Mattern I, Salnikov AV, Moldenhauer G, Wagner W, Diehlmann A, Saffrich R, Schubert M, Ho AD, Giese N, Buchler MW, Friess H, Buchler P, Herr I. VEGF expression by mesenchymal stem cells contributes to angiogenesis in pancreatic carcinoma. Br J Cancer. 2008; 99: 622-31.

26. Wang Y, Wan C, Gilbert SR, Clemens TL. Oxygen sensing and osteogenesis. Ann N Y Acad Sci. 2007; 1117: 1-11.

27. Ferrara N, Gerber HP, LeCouter J. The biology of VEGF and its receptors. Nat Med. 2003; 9: 669-76

28. Nikol S, Engelmann MG, Pelisek J, Fuchs A, Golda A, Shimizu M, Mekkaoui C, Rolland PH. Local perivascular application of low amounts of a plasmid encoding for vascular endothelial growth factor (VEGF165) is efficient for therapeutic angiogenesis in pigs. Acta Physiol Scand. 2002; 176: 151-9.

29. Elcin YM, Dixit V, Gitnick G. Extensive in vivo angiogenesis following controlled release of human vascular endothelial cell growth factor: implications for tissue engineering and wound healing. Artif Organs. 2001; 25: 558-65.

30. Carr AN, Howard BW, Yang HT, Eby-Wilkens E, Loos P, Varbanov A, Qu A, DeMuth JP, Davis MG, Proia A, Terjung RL, Peters KG. Efficacy of systemic administration of SDF-1 in a model of vascular insufficiency: support for an endothelium-dependent mechanism. Cardiovasc Res. 2006; 69: 925-35.

31. Tomanek RJ, Lotun K, Clark EB, Suvarna PR, Hu N. VEGF and bFGF stimulate myocardial vascularization in embryonic chick. Am J Physiol. 1998; 274: H1620-6.

32. Ziche M, Maglione D, Ribatti D, Morbidelli L, Lago CT, Battisti M, Paoletti I, Barra A, Tucci M, Parise G, Vincenti V, Granger HJ, Viglietto G, Persico MG. Placenta growth factor-1 is chemotactic, mitogenic, and angiogenic. Lab Invest. 1997; 76: 517-31.

33. Thurston G, Rudge JS, Ioffe E, Zhou H, Ross L, Croll SD, Glazer N, Holash J, McDonald DM, Yancopoulos GD. Angiopoietin-1 protects the adult vasculature against plasma leakage. Nat Med. 2000; 6: 460-3.

34. Fukuhara S, Sako K, Noda K, Zhang J, Minami M, Mochizuki N. Angiopoietin-1/Tie2 receptor signaling in vascular quiescence and angiogenesis. Histol Histopathol. 2010; 25: 387-96.

35. Qu D, Li J, Li Y, Gao Y, Zuo Y, Hsu Y, Hu J. Angiogenesis and osteogenesis enhanced by bFGF ex vivo gene therapy for bone tissue engineering in reconstruction of calvarial defects. J Biomed Mater Res A. 2011; 96: 543-51.

36. Street J, Bao M, deGuzman L, Bunting S, Peale FV, Jr., Ferrara N, Steinmetz H, Hoeffel J, Cleland JL, Daugherty A, van Bruggen N, Redmond HP, Carano RA, Filvaroff $\mathrm{EH}$. Vascular endothelial growth factor stimulates bone repair by promoting angiogenesis and bone turnover. Proc Natl Acad Sci U S A. 2002; 99: 9656-61.

37. Dai J, Rabie AB. VEGF: an essential mediator of both angiogenesis and endochondral ossification. J Dent Res. 2007; 86: 937-50.

38. Brandi ML, Collin-Osdoby P. Vascular biology and the skeleton. J Bone Miner Res. 2006; 21: 183-92.

39. Clarkin CE, Emery RJ, Pitsillides AA, Wheeler-Jones CP. Evaluation of VEGF-mediated signaling in primary human cells reveals a paracrine action for VEGF in osteoblast-mediated crosstalk to endothelial cells. J Cell Physiol. 2008; 214: 537-44.

40. Villars F, Guillotin B, Amedee T, Dutoya S, Bordenave L, Bareille R, Amedee J. Effect of HUVEC on human osteoprogenitor cell differentiation needs heterotypic gap junction communication. Am J Physiol Cell Physiol. 2002; 282: C775-85.

41. Guillotin B, Bourget C, Remy-Zolgadri M, Bareille R, Fernandez P, Conrad V, Amedee-Vilamitjana J. Human primary endothelial cells stimulate human osteoprogenitor cell differentiation. Cell Physiol Biochem. 2004; 14: 325-32.
42. Wu C, Zhou Y, Chang J, Xiao Y. Delivery of dimethyloxallyl glycine in mesoporous bioactive glass scaffolds to improve angiogenesis and osteogenesis of human bone marrow stromal cells. Acta Biomater. 2013;9:9159-68. 